\title{
Evaluation of the efficacy of triple therapy regimen for Helicobacter pylori eradication in gastrectomized patients with gastric adenocarcinoma
}

\author{
Jose Luiz Bertoli Neto ${ }^{1}$, Laercio Gomes Lourenço² ${ }^{2}$ Cícero Fernando Bertoli ${ }^{3}$, \\ Fábio Samsonowski Ulbrich ${ }^{4}$, Antoninho Ricardo $\mathrm{Sabbi}^{4}$, and Alexandre Galvão Bueno 5 \\ ${ }^{1}$ Department of Surgery, Fellow of Universidade Federal de São Paulo - Escola-Paulista de Medicina, São Paulo, Brazil \\ ${ }^{2}$ Department de GI Surgery of Universidade Federal de São Paulo - Escola Paulista de Medicina, São Paulo, Brazil \\ ${ }^{3}$ Institute of Gastroenterology and Surgery of Foz do Iguaçu, Brazil \\ ${ }^{4}$ Department of Oncology Surgery, Hospital Ministro Costa Cavalcanti, Foz do Iguaçu, Brazil \\ ${ }^{5}$ Institute of Pathology and Cytopathology, Cascavel, Brazil
}

\begin{abstract}
Background. The cause of cancer in the gastric stump is multifactorial, and Helicobacter pylori is one of these factors. Its eradication has been recommended; however, there are few studies about of $\boldsymbol{H}$. pylori eradication in gastrectomized patients.

Methods. Twenty gastrectomized patients with gastric adenocarcinoma and Roux-en-Y reconstruction (study group) infected by $\boldsymbol{H}$. pylori were compared with nongastrectomized patients (control group) also infected by $H$. pylori. The presence of $\boldsymbol{H}$. pylori was determined by the ultra-quick urease test and from a histological sample obtained by endoscopy. Both groups received the same triple therapy regimen.

Results. The rate of eradication of $H$. pylori in the study group was $90 \%$ and in the control group, it was $85 \%$. Sex, age, and postoperative time did not influence the rate of eradication.

Conclusion. There were no differences in the efficacy of $H$. pylori eradication between the two groups; therefore, the triple therapy regimen is effective for the eradication of $\boldsymbol{H}$. pylori in gastrectomized patients with a Roux-en-Y reconstruction.
\end{abstract}

Key words Helicobacter pylori $\cdot$ Eradication · Triple therapy · Gastric cancer

\section{Introduction}

The association between bacteria and gastric illness has been studied for a long time, with the participation of

Offprint requests to: J.L. Bertoli Neto

Rua Marechal Floriano Peixoto 735 Centro - Foz do Iguaçu

- Paraná Brazil - cep 85851-020

Received: April 3, 2006 / Accepted: July 4, 2006 the Helicobacter pylori in this association being discovered at the end of the last century by Marshall and Warren. Since then, studies have related H. pylori to several gastric illnesses, including gastric cancer [1,2]. The eradication of this bacteria is one of the subjects most studied in this regard [3,4]. Most of the published studies are about the eradication of $H$. pylori in nonoperated stomachs; however, there are few references to this eradication in gastrectomized patients; in particular, in those with a Roux-en-Y diversion [5]. As far as we know the efficacy of antibiotics for the eradication of $H$. pylori depends on several factors: the gastric $\mathrm{pH}$, the level of drug in the gastric mucosa, and acquired resistance [6]. These factors could explain some of the alterations that occur in the efficacy of $H$. pylori eradication when medications are used in patients with a partially removed stomach.

The cause of gastric stump cancer is multifactorial, and $H$. pylori seems to be one of these factors [7]. The eradication of $H$. pylori decreases the risk of the development of gastric stump cancer [8]. Therefore, it is considered of fundamental importance to carry out a clinical study that could verify the efficacy of a triple therapy regimen in gastrectomized patients. This study was carried out to compare the efficacy of $H$. pylori eradication with a triple therapy regimen (clarithromycin, amoxicillin, and lansoprazole) in gastrectomized and nongastrectomized patients, having as an hypothesis that there could be alterations in the efficacy of the treatment (due to the physiological and anatomical differences of the gastrectomized stomach), which could mean that a new therapeutic regimen would be needed. 


\section{Patients and methods}

\section{Patients}

Nongastrectomized patients constituted our control group (40 patients, 18 men [45\%] and 22 women [55\%], aged between 30 and 61 years; average, 45.5 years). The study group had a total of 20 patients who were submitted to subtotal gastrectomy with a Roux-en-Y diversion, due to gastric adenocarcinoma (12 men [60\%] and 8 women [40\%], aged between 45 and 72 years; average, 59 years. The patients in both groups were $H$. pyloripositive and presented symptomatology that justified the performance of upper gastrointestinal endoscopy (endoscopy). The criteria for exclusion for both groups were: use of anti-inflamatories, antibiotics, antiacids, and proton pump inhibitors within the previous 90 days, as well as patients who had gastric ulcer and/or were carriers of neoplasms. Patients who had received chemotherapy and/or radiotherapy in the previous 120 days were also excluded.

\section{Methods}

In both groups, H. pylori was identified by the ultraquick urease test and from a histological sample obtained by endoscopic biopsy examination; initially, there were four biopsies in each group. In the control group, a biopsy of the antrum and one of the corpus were submitted to the urease ultra-quick test in separate flasks, while the other two biopsies were examined histologically, also in separate flasks. In the study group, the procedure was the same, the only difference being the locations of the biopsies; one was taken from the corpus and the other from the fundus. The ultra-quick test was carried out in the endoscopy room. We used Giemsa and hematoxylin-eosin staining for the histological examination. Patients who presented with any positive tests were considered $H$. pylori carriers. The treatment for $H$. pylori was initiated as soon as the infection was detected; both groups followed a 7-day treatment schedule, taking lansoprazole $30 \mathrm{mg}$ (po), amoxicillin $1.0 \mathrm{~g}$ (po), and clarithromycin $500 \mathrm{mg}$ (po) every $12 \mathrm{~h}$.

Ninety days after the treatment was finished, the same approach for $H$. pylori identification was used; however, eight biopsies were taken from each group. In the control group, two biopsies were taken from the antrum and two from the corpus, and divided in two different flasks, for the urease ultra-quick test. The same approach was used for the histological examination. In the study group, the only difference was in the locations of the biopsies. The patients were considered cured when both tests showed no presence of the bacteria.
Statistical analysis was carried out using the $\chi^{2}$ test, Fisher's test, and the homogeneity test; $P$ was considered significant when the value was less than 0.05. Bio-estat 2.0 and Statistics 6.0 for Windows XP were the programs used for the analysis.

\section{Results}

In the control group, the urease ultra-quick test diagnosed the presence of $\mathrm{H}$. pylori in both the antrum and corpus of 32 patients $(80 \%)$, with eight false-negative film strips. The histological examination was positive for H. pylori in 36 patients $(90 \%), 26$ in the antrum and corpus and 8 only in the corpus, with four false-negative film strips. After treatment, 6 patients remained $H$. pylori-positive, showing an index of eradication of $85 \%$. Histologically, 2 were positive in the antrum and 4 in the corpus. The ultra-quick urease test showed four falsenegative film strips, diagnosing $H$. pylori in only 2 patients, both in the corpus.

In the study group, the urease ultra-quick test showed the presence of $H$. pylori in 16 patients $(80 \%), 12$ were simultaneously in the corpus and fundus and 3 were in the fundus and in the corpus, with four false-negative film strips. The histological examination was positive in 18 patients (90\%), 9 simultaneously in the corpus and fundus, 7 in the fundus, and 2 only in the corpus, showing two false-negative film strips.

After treatment, two patients remained $H$. pylori-positive, with an index of eradication of $90 \%$. Histological diagnoses showed $H$. pylori simultaneously in the corpus and fundus, with one only in the fundus and the other in the corpus. The ultra-quick urease test showed the presence of $H$. pylori in the fundus of one patient, with a false-negative slide. Comparison of the efficacies of eradication in the two groups did not show a statistically significant difference $(P=0.7068)$. Evaluating the influence of sex, the medication was efficient in $35 \%$ of men in the control group and in $55 \%$ of men in the study group. For women, the triple therapy was efficient for $50 \%$ in the control group and for $35 \%$ in the study group. Considering the sex of the patients, we found no significant difference either in the control group $(P=0.58)$ or in the study group $(P=0.63)$. According to the postoperative time, by classes, we also established no differences between the groups $(P=0.47)$. We established that the duration of the postoperative period was not relevant for $H$. pylori eradication in terms of the presented data.

In the analysis of the study group, subdivided into three classes according to the age, we were able to verify that it was homogeneous and that efficacy did not differ by these classes $(P=0.11)$. 


\section{Discussion}

Rather than using a breath test, we chose to use the ultra-quick urease test and histological examination for the diagnosis of $H$. pylori. The ultra-quick urease test has high sensitivity and specificity [9], as does the histological examination [10-12], and this was carried out using two diferent staining methods, hematoxylin-eosin and Giemsa, decreasing the chances of false-negative results $[11,12]$. The use of two approaches for the detection of $H$. pylori was designed to make the diagnosis accurate, because, after treatment, there is a lower bacterial population, diminishing the sensitivity of the diagnostic approaches [13]. The period of 90 days between $H$. pylori eradication and the control test seemed to be satisfactory [14], because an 8-week period or less is subject to false-negative results [15]. Prior studies have shown that the predominance and the density of $H$. pylori depend on the gastric location [16], indicating the importance of the number and locations of the biopsies [17]. Our choice of four biopsies for diagnosis and eight for control was based on proof found in the literature; however, we could not find standardization for biopsies in gastrectomized patients. The prevalence of $H$. pylori in Brazil varies form $62.3 \%$ to $85 \%$ [18]. In the control group, we found a prevalence of $83.3 \%$, which is in accordance with the literature. We believe that this congruity occurred because the control group was of the same socioeconomic status as the patients in the literature. The prevalence of $H$. pylori in gastrectomized patients varies according to several factors; such as the postoperative period, the technique employed, and the age of the patient [19-22]. The prevalence of $H$. pylori in Billroth I gastrectomized patients varies from $36.9 \%$ to $80.3 \%[5,19,20]$, while that in Billroth II gastrectomized patients varies from $0 \%$ to $58 \%$ [19,20,23]. There are few references in the literature concerning H. pylori and Roux-en-Y diversion; however, this technique could provide a high prevalence rate having in mind that biliary reflux is damaging to $H$. pylori [20-23]. The study group presented an $H$. pylori prevalence of $71.4 \%$. We believe that this prevalence was not bigger due to the short postoperative period and to possible treatment prior to the surgery. Guidelines for the eradication of $H$. pylori have been recommended in Brazil [24]; however, it was not the purpose of this study to determine the efficacy of a treatment, but to compare two patient groups. In Brazil, it has been shown that therapeutic efficacies vary from $85.1 \%$ to $89 \%$ in different regions depending on socioeconomic levels [25]. In the present study, the eradication rate in the control group was $85 \%$, coinciding with previous values. The literature shows a broad range regarding rates of eradication of $H$. pylori in gastrectomized patients; however, the numbers refer to patients operated by several tech- niques, all regarded in the same group [5,7,22]. The patients in our study group were submitted to the same surgical technique; from our point of view, this led to a homogeneous study group. The eradication rate in this group was $90 \%$. Thus, comparing the efficacy of the treatment in our two groups, no statistically significant difference was shown. When evaluating the influence of sex on the treatment there was no statistically significant influence of this factor on the results. The duration of the postoperative period also did not show statistical relevance. We conclude that the triple treatment is efficient for the eradication of $H$. pylori in gastrectomized patients with a Roux-en-Y diversion. We believe that new studies must be carried out with these operated patients to evaluate recurrence of the infection and the resistance of the bacterium.

\section{References}

1. Sugiyama A, Maruta F, Ikeno T, Ishida K, Kawasaki S, Katsuyama T, et al. Helicobacter pylori infection enhances Nmethyl-N-nitrosourea-induced stomach carcinogenesis in Mongolian gerbils. Cancer Res 1998;58:2067-9.

2. Uemura N, Okamoto S, Yamamoto S, Matsumura N, Yamaguchi S, Yamakido M, et al. Helicobacter pylori infection and the development of gastric cancer. N Engl J Med 2001;345:784-9.

3. The European Helicobacter pylori Study Group. Current European concepts in the management of Helicobacter pylori infection. The Maastricht Consensus Report. Gut 1997;41:8-13.

4. Miwa H, Nagahata A, Sato K, Ohkura R, Murai T, Shimizu H, et al. Efficacy of 1 week omeprazole or lansoprazole-amoxicillinclarithromycin therapy for Helicobacter pylori infection in the Japanese population. J Gastroenterol Hepatol 1999;14:317-21.

5. Rino Y, Imada T, Shiozawa M, Takahashi M, Fukuzawa K, Hasuo $\mathrm{K}$, et al. Helicobacter pylori of the remnant stomach and its eradication. Hepatogastroenterology 1999;46:2069-73.

6. Megraud F, Cayla R, Lamouliatte H, Bouchard S, Darmaillac V. Surveillance of Helicobacter pylori resistance to macrolides and nitroimidazole compounds at a nation level. Am J Gastroenterol 1994;89:1368-9.

7. Matsukura N, Tajiri T, Kato S, Togashi A, Masuda G, Fujita I, et al. Helicobacter pylori eradication therapy for the remnant stomach after gastrectomy. Gastric Cancer 2003;6:100-7.

8. Saito K, Arai K, Mori M, Kobayashi R, Ohki I. Effect of Helicobacter pylori eradication on malignant transformation of gastric adenoma. Gastrointest Endosc 2000;52:27-32.

9. Thillainayagam AV, Arvind AS, Cook RS, Harrison IG, Tabaqchali S, Farthing MJG. Diagnostic efficiency of an ultrarapid endoscopy room test for Helicobacter pylori. Gut 1991; 32:467-9.

10. Morais M, Macedo EP, Silva MR Jr, Rohr MRS, Ferraz MLG, Castro RRO, et al. Comparison of invasive tests for Helicobacter pylori infection diagnosis. Arq Gastroenterol 1997;34:207-11.

11. Cohen H, Laine LA. Endoscopic methods for the diagnosis of Helicobacter pylori. Aliment Pharmacol Ther 1997;11 (Suppl 1): 3-10.

12. Howden $\mathrm{CW}$, Hunt RH. Guidelines for the management of Helicobacter pylori infection. Am J Gastroenterol 1998;93:23308.

13. Queiroz DMM. Invasive tests for Helicobacter pylori infection diagnosis. Arq Gastroenterol 1997;34:205-6.

14. Katelaris PH, Jones DB. Testing for Helicobacter pylori infection after antibiotic treatment. Am J Gastroenterol 1997;92:1245-7. 
15. El-Zimaty HMT, Al-Assi MT, Genta RM, Graham DY. Confirmation of successful therapy of Helicobacter pylori infection: number and site of biopsies or a rapid urease test. Am J Gastroenterol 1995;90:1962-4.

16. Carvalhaes A, Magalhães AFN. Pesquisa do Helicobacter pylori (in Portuguese). In: Carvalhaes, Magalhães AFN, editors. Endoscopia digestiva diagnóstica e terapêutica. 4a ed. Rio de Janeiro: Revinter: 2005. pp. 311-5.

17. El-Zimaty HMT, Graham DY. Evaluation of gastric mucosal biopsy site and number for identification of Helicobacter pylori or intestinal metaplasia: role of the Sydney system. Hum Pathol 1999;30:72-7.

18. Coelho LGV, Zarqueta S, and representatives of the Brazilian Federation of Gastroenterology and Brazilian Nucleus for the Study of Helicobacter. Second Brazilian Consensus Conference on Helicobacter pylori Infection. Arq Gastroenterol 2005;42:12832.

19. Onoda N, Maeda K, Sawada T, Wakasa K, Arakawa T, Hirakawa K. Prevalence of Helicobacter pylori infection in gastric remnant after distal gastrectomy for primary gastric cancer. Gastric Cancer 2001:4:87-92.

20. Tomtitchong P, Onda M, Matsukura N, Tokunaga A, Kato S, Matsuhisa T, et al. Helicobacter pylori infection in remnant stom- ach after gastrectomy: with special reference to the difference between Billroth I and II anastomosis. J Clin Gastroenterol 1998; 27:S154-8.

21. O'Connor HJ. Helicobacter pylori and Roux-en-Y diversion. Eur J Gastroenterol Hepatol 2004;16:361-2.

22. Kim ES, Park DK, Hong SH, Chung MG, Kwon OS, Kim SS, et al. Helicobacter pylori infection in the remnant stomach after radical subtotal gastrectomy. Korean J Gastroenterol 2003;42: $108-14$.

23. Katsube T, Ogawa K, Hamaguchi K, Murayama M, Konnno S, Shimakawa T, et al. Prevalence of Helicobacter pylori in the residual stomach after gastrectomy for gastric cancer. Hepatogastroenterology 2002;49:128-32.

24. Coelho LGV, León-Barúa R, Quigley EMM, and representatives of the Latin-American National Gastroenterological Societies affiliated with the Inter-American Association of Gastroenterology (AIGE). Latin-American consensus conference on Helicobacter pylori infection. Am J Gastroenterol 2000;95:268891.

25. Bellelis P, Samano EST, Nunes RC, Ribeiro LM, Chehter EZ, Catapani WR. Efficacy of a triple terapy for Helicobacter pylori eradication in a well-developed urban area in Brazil. Sao Paulo Med J 2004;122:73-5. 